\title{
Comparative thermal characteristics and fatty acid composition of mono- and diacylglycerols of lard and some commercial emulsifiers
}

\begin{abstract}
A study was carried out to compare the fatty acid composition and thermal profiles of monoand diacylglycerols of six commercial emulsifiers, coded as E1, E2, E3, E4, E5, and E6, purchased from different manufacturers with those of the same derived from lard. The lipid extraction from the commercial emulsifiers was done using the Soxhlet method and the isolation of individual mono- and diacylglycerols was carried out using a column chromatographic method. The isolated partial acylglycerols of lard and individual emulsifiers were subjected to fatty acid analysis by gas chromatography and thermal analysis by differential scanning calorimetry. A clear distinction between lard-based emulsifier and the commercial emulsifiers was achieved by the application of principal components analysis to the fatty acid data. According to thermal analysis, mono- and diacylglycerols of lard displayed significant differences from those of commercial mono- and diacylglycerols with respect to the thermal transitions in the cooling curves. This study concluded that partial acylglycerols of none of the commercial emulsifiers employed in this study displayed similarity to those of lard-based emulsifiers.
\end{abstract}

Keyword: Commercial emulsifiers; Diacylglycerol; Fatty acid; Monoacylglycerol; Principal components analysis; Thermal analysis 